BIOCYT Biología, Ciencia y Tecnología, 13: 957-974, 2020.

ISSN: 2007-2082

DOI: http://dx.doi.org/10.22201/fesi.20072082e.2020.13.77221

http://revistas.unam.mx/index.php/biocyt

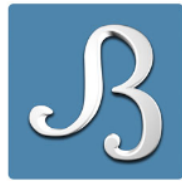

Publicada en la Facultad de Estudios Superiores Iztacala, Universidad Nacional Autónoma de México

\title{
CRECIMIENTO Y ASPECTOS TROFICOS DEL PEZ LEON ROJO PTEROIS VOLITANS (SCORPAENIFORMES: SCORPAENIDAE) EN EL PARQUE NACIONAL SISTEMA ARRECIFAL VERACRUZANO (PNSAV), VERACRUZ, MÉXICO
}

\author{
GROWTH AND TROPHIC ASPECTS OF THE RED LIONFISH \\ PTEROIS VOLITANS (SCORPAENIFORMES: SCORPAENIDAE) IN \\ THE VERACRUZANO ARRECIFAL SYSTEM NATIONAL PARK \\ (VASNP), VERACRUZ, MEXICO
}

\author{
Mariajosé Xhail Amador-Núñez y Ángel Morán-Silva2 ${ }^{2 \times]}$ \\ Laboratorio de Crustáceos, Facultad de Estudios Superiores Iztacala, Universidad Nacional Autónoma de \\ México, Av. de los Barrios 1, Los Reyes Iztacala, Tlalnepantla, Estado de México, C.P. 54090, México \\ ${ }^{1}$ xhailbvb@gmail.com , ${ }^{2}$ cygamoran@gmail.com \\ Autor de correspondencia/corresponding author $\square$
}

\begin{abstract}
The lionfish Pterois volitans, is an predator in coral system, whose diet is composed of small fish and crustaceans, impacting species of ecological and commercial importance. P. volitans is an exotic species in the Gulf of Mexico with high colonization capacity. The present study describes its population structure, type of growth and trophic dynamics. 97 organisms of the PNSAV were obtained, 64 females (185 $\pm 6,7 \mathrm{~mm} \mathrm{LP} ; 257,6 \pm 27,8 \mathrm{~g})$ and 22 males $(183 \pm 15,8 \mathrm{~mm}$ of LP; $273,5 \pm 62,6 \mathrm{~g})$. Whit the Battacharya model, four size classes were obtained for females $(15,3 ; 25,3 ; 28,2$, and 31,8 $\mathrm{cm}$ ) and six for males $(9,87 ; 17,95 ; 22,95 ; 26,95 ; 30,95$ and 33,95), being the size class 1 for both sexes the most abundant. The length-weight relationship was of the potential type $\left(r^{2}=0,9643, b=3,1574\right.$ females, and $r^{2}=0,9843$ and $b=3,1089$ males), and growth of allometric type for both sexes. The growth model that best adjusted to the data was that of Gompertz, $\left(\mathrm{L}_{\infty}=324 \mathrm{~mm}, \mathrm{k}=0,99, \mathrm{t}_{0}=0,694\right.$ females and $\mathrm{L}_{00}=379,4, \mathrm{k}=0,468, \mathrm{t}_{0}=0,719$ males). The families of fish identified in the stomach contents were Ophididae, Serranidae, Haemulidae, Pomacentridae, Beloniidae, Achiridae, and crustaceans Portunidae, Penaeidae, Squillidae and Diogenidae. Being Haemulon aurolineatum (Haemulidae) and Xiphopenaeus kroyeri (Penaeidae) the most important prey. The presence of lionfish in the PNSAV is recent, so the information presented in this research will allow us to understand its population dynamics and its adaptation to the PNSAV, contributing to its management and control.
\end{abstract}

Key words: coral reef; diet; growth; Gulf of Mexico; gut content; invasive species.

Manuscrito recibido el 10 de julio de 2020, aceptado el 06 de septiembre de 2020. 


\section{RESUMEN}

El pez león Pterois volitans es un depredador importante en sistemas coralinos, cuya dieta está compuesta por pequeños peces y crustáceos impactando a especies de importancia ecológica y comercial. $P$. volitans es una especie exótica en el golfo de México con alta capacidad de colonización, el presente estudio describe su estructura poblacional, tipo de crecimiento y dinámica trófica. Se obtuvieron 97 organismos en el PNSAV, 64 hembras $(185 \pm 6.7 \mathrm{~mm}$ de LP; $257.6 \pm 27.8 \mathrm{~g})$ y 22 machos ( $183 \pm 15.8 \mathrm{~mm}$ de LP; $273.5 \pm 62.6 \mathrm{~g})$. Con el modelo de Battacharya se obtuvieron cuatro clases de talla para hembras $(15.3,25.3,28.2$ y $31.8 \mathrm{~cm})$ y seis para machos $(9.87,17.95,22.95,26.95$, 30.95 y 33.95), siendo la clase de talla 1 para ambos sexos la más abundante. La relación talla-peso fue de tipo potencial $\left(\mathrm{r}^{2}=0.9643, \mathrm{~b}=3.1574\right.$ hembras y $\mathrm{r}^{2}=0.9843, \mathrm{~b}=3.1089$ machos $)$, y crecimiento de tipo alométrico para ambos sexos. El modelo de crecimiento que mejor se ajustó a los datos fue el de Gompertz, (hembras $\mathrm{L}_{00}=324 \mathrm{~mm}, \mathrm{k}=0.99, \mathrm{t}_{0}=0.694 ; \mathrm{y}$ machos $\mathrm{L}_{00}=379.4, \mathrm{k}=0.468, \mathrm{t}_{0}=0.719$ ). Las familias de peces identificadas en contenido estomacal fueron Ophididae, Serranidae, Haemulidae, Pomacentridae, Beloniidae y Achiridae, y de crustáceos fueron Portunidae, Penaeidae, Squillidae y Diogenidae. Siendo Haemulon aurolineatum (Haemulidae) y Xiphopenaeus kroyeri (Penaeidae) las presas más importantes. La presencia del pez león en el PNSAV es reciente, por lo que la información presentada en la presente investigación permitirá comprender su dinámica poblacional y su adaptación al PNSAV, contribuyendo a su manejo y control.

Palabras clave: arrecife de coral; contenido estomacal; crecimiento; dieta; especie invasora; golfo de México.

\section{INTRODUCCIÓN}

El pez león rojo (Pteoris volitans Linnaeus, 1758) se distribuye de forma natural en la región del Indo-Pacífico en aguas tropicales y arrecifes coralinos, siendo organismos solitarios e inmóviles (Schultz, 1986; Kuiter y Tonozuca, 2001). Se caracteriza por poseer barras y bandas de color marrón rojizo, los adultos pueden tener manchas blancas a lo largo de la línea lateral, cuentan con 13 espinas en la aleta dorsal, 14 rayos pectorales dispuestos como abanicos y con tres espinas y de seis a siete radios en la aleta anal, pudiendo presentar un tentáculo por encima de los ojos (Kuiter y Tonozuca, 2001).

Los organismos de esta especie pueden alcanzar $390 \mathrm{~mm}$ de longitud máxima (AguilarPerera et al., 2013), son peces que presentan de manera general, crecimiento de tipo alométrico, resultado de un acelerado crecimiento en longitud en las primeras etapas de vida, sin embargo en una etapa avanzada de su ciclo de vida disminuye la velocidad de crecimiento, en tanto que el organismo continúa ganando peso; debido a lo anterior el pez león alcanza la etapa reproductiva en tan solo un año (Barbour et al., 2011; Sabido-Itzá et al., 2016). El pez león ha sido identificado como uno de los principales depredadores en sistemas coralinos, ya que cazan pequeños peces (incluyendo organismos de su propia especie), camarones y cangrejos (Fishelson, 1977; VillaseñorDerbez y Herrera-Pérez, 2014). De acuerdo con Morris y Akins (2009) se han determinado 41 especies diferentes de teleósteos en contenidos estomacales de ésta especie, resaltando la presencia de peces de importancia tanto ecológica como comercial.

Desde su introducción accidental en el golfo de México, $P$. volitans ha ido incrementando su distribución llegando hasta el mar caribe, debido posiblemente a que las condiciones ambientales características de ésta área suelen ser mejores que en las de su hábitat nativo (Darling et al., 2011). El primer avistamiento de pez león en aguas mexicanas fue registrado en Puerto Progreso, Yucatán en el año 2009, reportando un espécimen de $137 \mathrm{~mm}$ de longitud total (LT) (Aguilar-Perera et al., 2010). En tanto que, el primer registro del pez león en el Parque Nacional Sistema Arrecifal 
Veracruzano (PNSAV) fue a través de un registro fotográfico en diciembre de 2011, subsecuentemente en enero de 2012 se registró el avistamiento de otro organismo en el PNSAV, presentando $185 \mathrm{~mm}$ de LT (Santander-Monsalvo et al., 2012). Sumado a los anteriores reportes, Wakida-Kusonoki y Amador (2015), registraron por primera vez la presencia de P. volitans en las costas del estado de Tabasco, en julio de 2013 y 2014.

Pterois volitans es considerada una especie exótica dentro del golfo de México, ya que se encuentra fuera de su área de distribución natural y presenta una alta capacidad de colonización (Edelist et al., 2013; Sabido-Itzá et al., 2016). De acuerdo con Lugo-Castro et al. (2018), las especies exóticas son capaces de modificar la cadena trófica y los ecosistemas donde se establecen, como las áreas marinas protegidas (AMP) las más vulnerables ante invasiones, identificándose como uno de sus efectos el impacto en el reclutamiento de peces arrecifales (Albins y Hixon, 2008), aunado al impacto que produce en especies de importancia ecológica y comercial por el consumo de tallas juveniles (Morris y Akins, 2009).

Por lo anterior y tomando en cuenta la importancia ecológica de los ecosistemas del sureste de México, muchos de los cuales son considerados como regiones prioritarias para la conservación, resulta importante el conocimiento de los principales aspectos de la biología de esta especie como la estructura poblacional, tipo de crecimiento, y su relación con la dinámica trófica, con el objetivo de establecer pautas de manejo que permitan el control de $P$. volitans en el golfo de México.

\section{MATERIALES Y MÉTODOS}

El PNSAV está constituido por dos de los más complejos e importantes grupos de arrecifes en el golfo de México (Salas-Pérez y Granados-Barba, 2008), con una extensión de 52,239 hectáreas (DOF, 1992). Ubicado frente al puerto de Veracruz y la localidad de Antón Lizardo, cuenta con un grupo de 11 arrecifes localizados al frente del puerto de Veracruz-Boca del Río y otro grupo de 12 arrecifes localizados frente a Antón Lizardo; ambos grupos divididos por el río Jamapa y delimitados al norte por el río La Antigua y al sur por el río Papaloapan (Salas-Pérez y GranadosBarba, 2008), los cuales vierten una gran cantidad de sedimentos (Krutak, 1997) (Fig. 1).

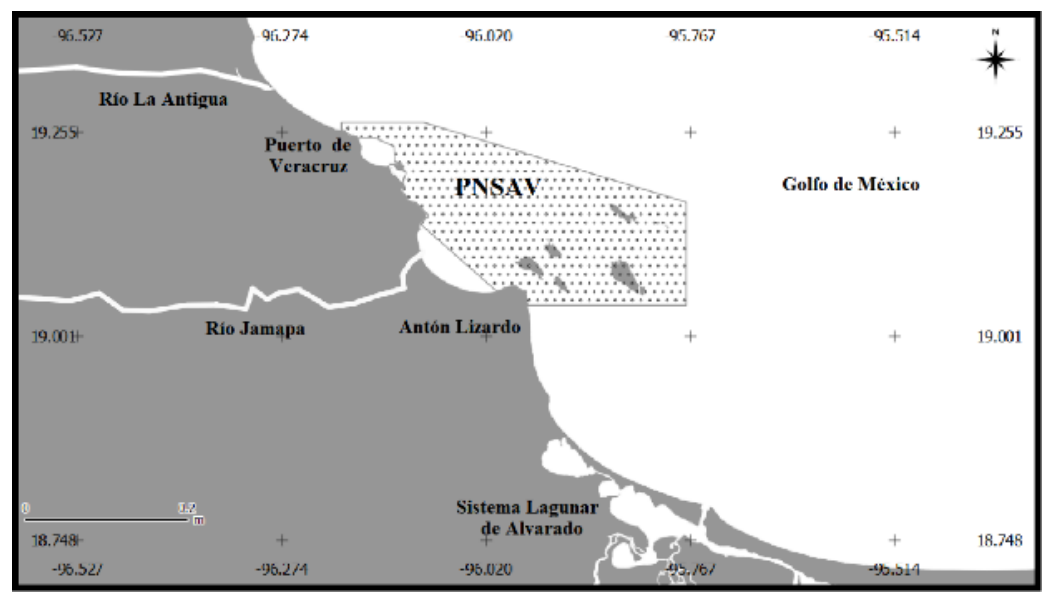

Fig 1. Localización del Parque Nacional Sistema Arrecifal Veracruzano, Veracruz, México. Fig 1. Location of National Park Sistema Arrecifal Veracruzano, Veracruz, México. 
Se obtuvieron organismos de capturas comerciales en la comunidad de Antón Lizardo en septiembre y noviembre de 2018; en el mes de marzo de 2019, se obtuvieron peces mediante el apoyo de la Comisión Nacional de Áreas Naturales Protegidas (CONANP). Todos los organismos fueron capturados dentro del polígono del PNSAV. Las muestras se depositaron en bolsas de polietileno y fueron resguardadas en congelador en el Instituto de Ciencias Marinas y Pesquerías de la Universidad Veracruzana, para su posterior procesamiento.

\section{Trabajo de laboratorio}

El material biológico fue trasladado al Laboratorio de Crustáceos de la UNAM-FES Iztacala para su procesamiento. Los organismos fueron descongelados, se obtuvieron los estómagos y se fijaron en formol al 10\% (Olaya-Nieto et al., 2009).

A cada organismo se le asignó un número de serie correspondiente a la fecha de captura. Los organismos fueron identificados a través de la obtención de datos merísticos (formula radial) de aletas dorsal y anal, siguiendo el criterio de Schultz (1986).

Se obtuvieron la longitud total (LT) y longitud patrón (LP) con la ayuda de un ictiómetro con precisión de $1 \mathrm{~mm}$. El peso total se obtuvo mediante una balanza granataria digital, con una precisión de $0.01 \mathrm{~g}$. Para el análisis de tallas se utilizó la longitud patrón de cada organismo.

\section{La relación longitud-peso fue representada por la ecuación}

Donde:

$\mathrm{W}=\mathrm{a} * \mathrm{Lb}$ (Ricker, 1975).

$\mathrm{W}=$ peso total del pez (expresado en gramos).

$\mathrm{L}=$ longitud patrón del pez (expresado en milímetros).

$\mathrm{a}=$ intersección.

$\mathrm{b}=$ pendiente de la recta.

Los valores de " $b$ " fueron evaluados con una prueba de $\mathrm{T}$ de Student, para determinar si existe una diferencia con la isomería ( $b=3$ ) (Sabido-Itzá et al., 2016).

Para conocer el grado de bienestar o condición somática de la especie en relación al medio invadido y la conveniencia del nuevo ambiente para esta especie, se calculó el factor de condición, expresado por la ecuación

Donde:

$$
\mathrm{K}_{\mathrm{N}}=\mathrm{M}_{\mathrm{T}} *\left(\mathrm{aL}_{\mathrm{T}}^{\mathrm{b}}\right)^{-1} \text { (Toledo-Hernández et al., 2014). }
$$

$$
\begin{aligned}
& \mathrm{M}_{\mathrm{T}}=\text { peso húmedo }(\mathrm{g}) . \\
& \mathrm{L}_{\mathrm{T}}=\text { longitud patrón }(\mathrm{mm}) . \\
& \mathrm{a}=\text { ordenada al origen. } \\
& \mathrm{b}=\text { pendiente de regresión. }
\end{aligned}
$$

Para obtener el agrupamiento de los datos de LP y la obtención de las clases modales se utilizó el modelo de Bhattacharya (1967) con ayuda del programa Excel (2013)

Para describir el crecimiento que presenta la especie, se empleó el modelo de crecimiento de Gompertz (1825), en este modelo la longitud se representa gráficamente en función de la edad, obteniendo una curva de tipo sigmoidea que describe un crecimiento inicial rápido, seguido de una 
desaceleración conforme al tiempo (Ecuación 1).

$N(t)=A e^{-B e-K t} \quad$ Ecuación 1

Dónde:

$\mathrm{N}(\mathrm{t})$ = es el tamaño de la población al tiempo " $\mathrm{t}$ "

$\mathrm{A}=$ es el valor del máximo crecimiento y determina un punto de inflexión en $\mathrm{A} / \mathrm{e}$

$\mathrm{B}=$ número positivo que desplaza el modelo a la izquierda o derecha

$\mathrm{K}=$ es la tasa intrínseca de crecimiento.

Para el análisis de la dinámica trófica se obtuvieron los tractos digestivos de cada organismo, se pesaron en una balanza granataria digital, se vació el contenido de los mismos y se volvieron a pesar. Así mismo se obtuvo el volumen del contenido estomacal con los tractos digestivos llenos y vacíos por el método de desplazamiento, en una probeta de $10 \mathrm{ml}$ (Korschgen, 1980). Con la información obtenida se realizó el análisis del contenido estomacal y hábitos alimenticios utilizando los métodos descritos por Yáñez-Arancibia (1986):

a) Numérico:

Donde:

$$
\mathrm{N}=\mathrm{n}(100) / \mathrm{N}
$$

$\mathrm{n}$ = suma de los elementos de un alimento en todos los estómagos.

$\mathrm{N}$ = suma de los elementos de los alimentos en todos los estómagos.

b) Gravimétrico:

Donde:

$$
\mathrm{P}=\mathrm{p}(100) / \mathrm{P}
$$

$\mathrm{p}$ = suma del peso de cierto alimento en todos los estómagos.

$\mathrm{P}$ =suma del peso del contenido estomacal de todos los estómagos.

c) Frecuencia:

Donde:

$$
\mathrm{F}=\mathrm{ne}(100) / \mathrm{NE}
$$

ne $=$ número de estómagos con un tipo de alimento.

$\mathrm{NE}=$ número de estómagos no vacíos examinados.

La identificación de los ítems alimenticios se llevó a cabo hasta donde fue posible debido al grado de digestión del alimento. El cálculo del valor de importancia de cada presa es dependiente de los factores descritos anteriormente, y se obtuvo a través del Índice de Importancia Relativa (IIR) (Pinkas et al., 1970):

Donde:

$$
\mathrm{IIR}=\mathrm{F}(\mathrm{N}+\mathrm{W})
$$

$\mathrm{F}=$ porcentaje de la frecuencia de ocurrencia.

$\mathrm{N}=$ porcentaje numérico.

$\mathrm{W}=$ porcentaje de peso.

\section{RESULTADOS}

\section{Relación talla-peso}

La relación talla-peso fue calculada con los datos de LP y peso neto (W) para machos (22 organismos) y hembras (64 organismos). Debido a que algunos organismos presentaron daño en el pedúnculo y aletas caudales, a causa del arpón hawaiano utilizado en el arte de pesca, y a que solo 86 de ellos pudieron ser identificados sexualmente, se utilizaron las LP de 86 organismos para este análisis. Los datos se ajustaron de mejor manera a una curva de tipo potencial con un coeficiente de correlación de $\mathrm{r}^{2}=0.9843$ y una pendiente de $\mathrm{b}=3.1089$ para machos (Fig. 3) y $\mathrm{r}^{2}=0.9638$ con una 
pendiente de $b=3.1574$ (Fig. 2) para hembras, presentando crecimiento de tipo alométrico positivo para ambos sexos $(t=2.0257 \mathrm{E}-4, P<0.05)$ y $(t=2.81 \mathrm{E}-14, P<0.05)$ respectivamente.

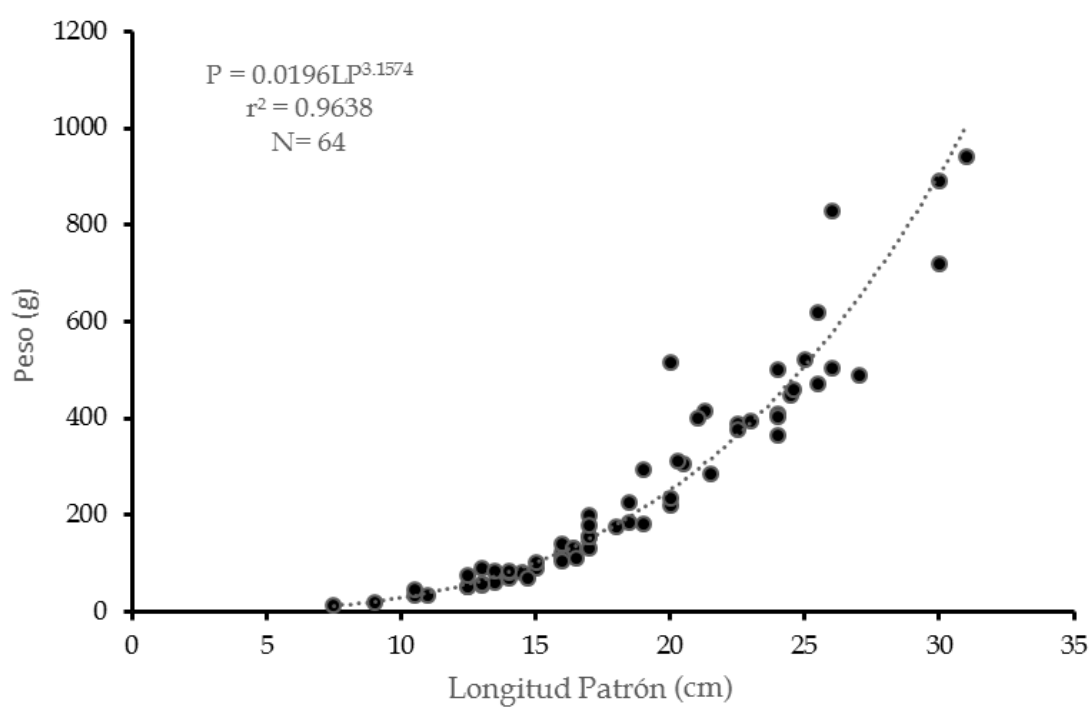

Fig. 2. Relación entre la longitud patrón y peso de hembras de P. volitans en el PNSAV, Veracruz, México. Fig. 2. Length- weight relationship of P. volitans females in the VASNP, Veracruz, Mexico.

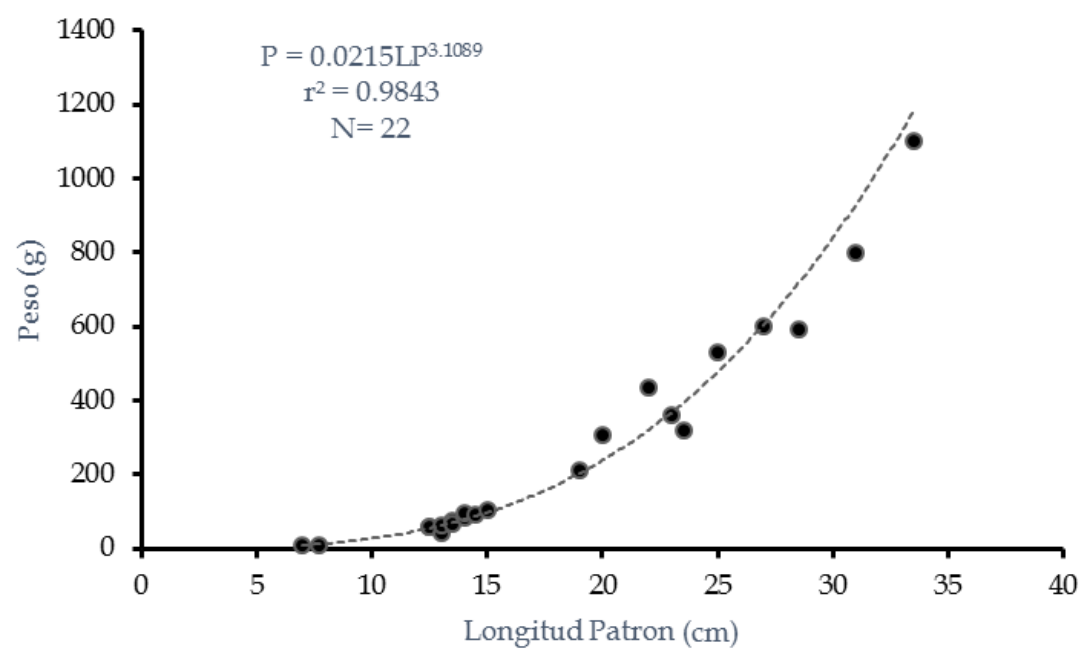

Fig. 3. Relación entre la longitud patrón y peso de machos de P. volitans en el PNSAV, Veracruz, México.

Fig. 3. Length- weight relationship of $P$. volitans males in the VASNP, Veracruz, Mexico. 


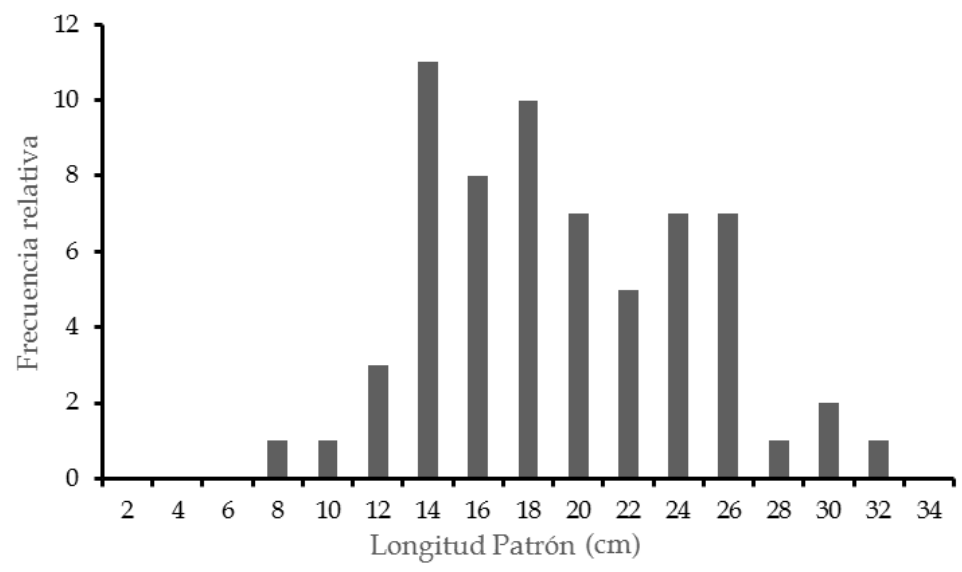

Fig. 4. Distribución tallas de hembras de P. volitans capturados en el PNSAV, Veracruz, México. Fig 4. Size distribution of P. volitans females, captured in the VASNP, Veracruz, Mexico.

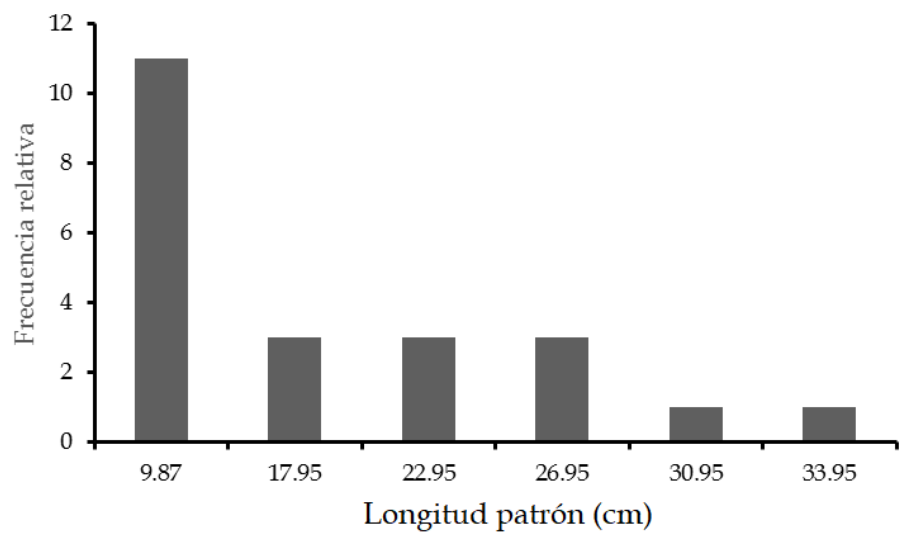

Fig. 5. Distribución tallas de machos de P.volitans capturados en el PNSAV, México. Fig 5. Size distribution of P. volitans males, captured in the VASNP, Veracruz, Mexico.

\section{Crecimiento}

Se obtuvieron cuatro clases de edad para hembras (Fig. 6), el grupo modal número 1 es el que presentó una mayor abundancia $(\mathrm{n}=46)$ en el intervalo de 7.5 y $21.8 \mathrm{~cm}$ de $\mathrm{LP}$, representando el $71.87 \%$ (Fig. 6), le siguió el grupo modal $2(\mathrm{n}=15)$ con tallas entre 22 y $27 \mathrm{~cm}$, con un $23.43 \%$ de representación, seguido por el grupo modal $3(n=2)$ que comprendió tallas entre 27.5 y $30 \mathrm{~cm}$, con una representación de $3.12 \%$, y finalmente, el grupo modal 4 representado por un organismo dentro del intervalo de tallas de 31 y $32.5 \mathrm{~cm}, 1.56 \%$ de la muestra.

En machos se estimaron seis clases de talla (Fig. 7). La clase de talla número 1 fue la que presentó mayor abundancia ( $\mathrm{n}=11)$, entre los 89 y $149 \mathrm{~mm}$, representando el 50\% de la muestra. Las clases de talla 2, 3 y $4(\mathrm{n}=3)$ presentaron intervalos de talla de 169 a $209 \mathrm{~mm}, 229$ a $249 \mathrm{~mm}$ y 269 a $289 \mathrm{~mm}$ respectivamente, con $13.63 \%$ de la abundancia para cada una, lo que suma $40.90 \%$. Finalmente los grupos modales 5 y 6 fueron los que presentaron un menor porcentaje $(4.54 \%)$ y comprenden los intervalos de talla de 309 a $329 \mathrm{~mm}$ y mayores a $349 \mathrm{~mm}$, respectivamente. 


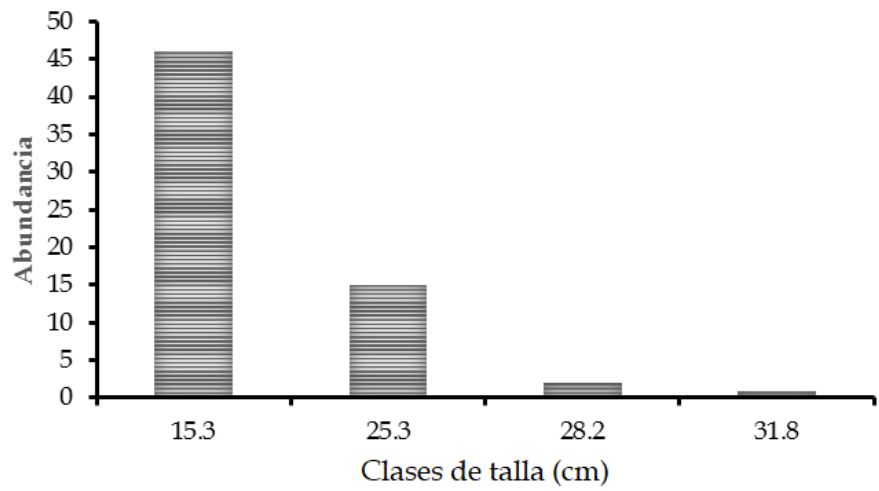

Fig. 6. Clases de tallas calculadas para hembras de P. volitans en el PNSAV, Veracruz, México.

Fig. 6. Age classes of P. volitans females in the VASNP, Veracruz, Mexico.

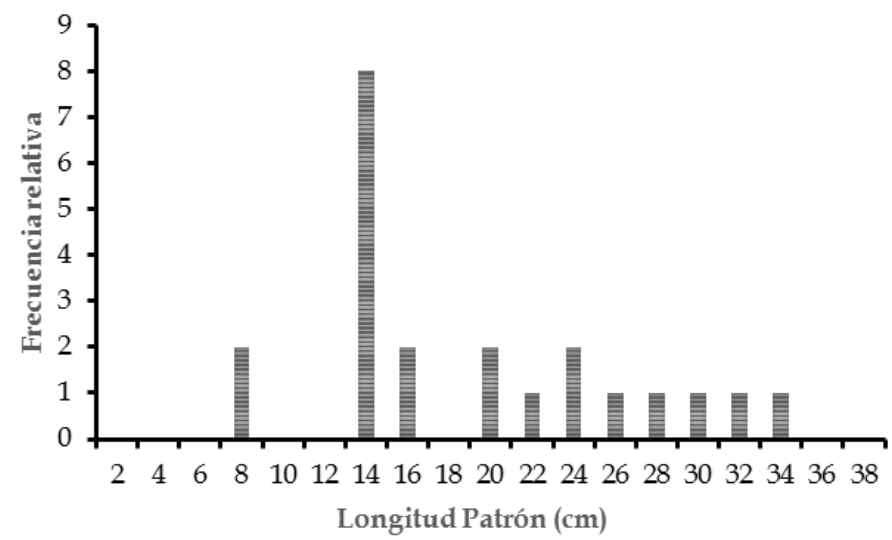

Fig. 7. Clases de tallas calculadas para machos de P. volitans en el PNSAV, Veracruz, México. Fig. 7. Age classes of P. volitans males in the VASNP, Veracruz, Mexico.

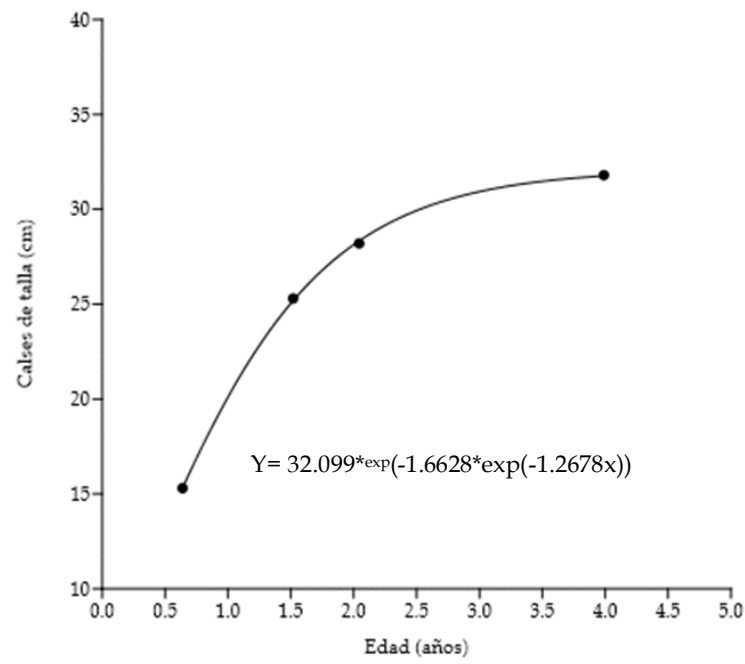

Fig. 8. Curva del modelo de crecimiento de Gompertz para hembras de P. volitans en el PNSAV, Veracruz, México. Fig. 8. Gompertz growth model curve for females P. volitans in the VASNP, Veracruz, Mexico. 
Las curvas del modelo de crecimiento se generaron con valores de L $\infty$ (324 mm),k (0.99) (Fig. 8) y $\mathrm{t}_{0}(-0.694)$ para hembras, y $\mathrm{L}_{00}(379.4 \mathrm{~mm}), \mathrm{k}(0.468)$ y $\mathrm{t}_{0}(-0.719)$ para machos (Fig. 9).

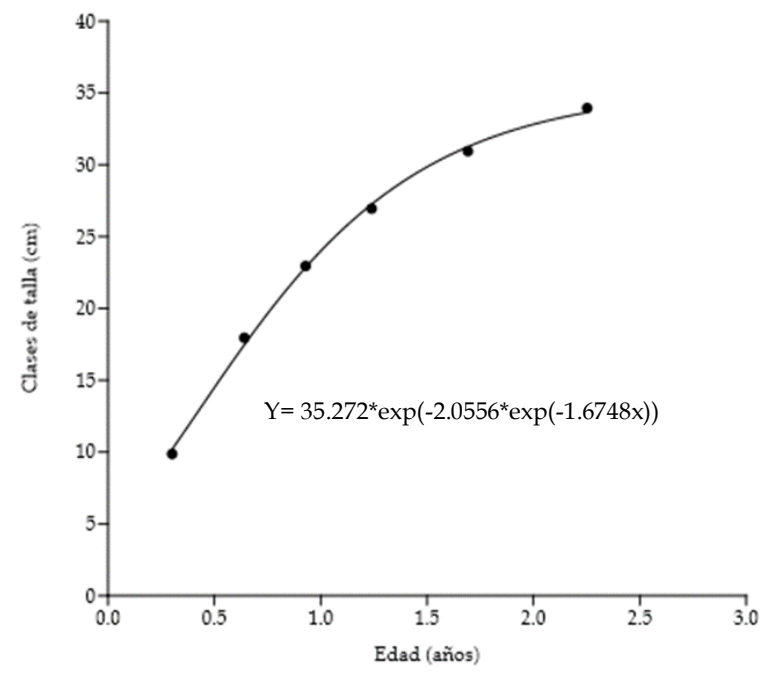

Fig. 9. Representación del modelo de crecimiento de Gompertz para machos de P. volitans en el PNSAV, Veracruz, México.

Fig. 9. Gompertz growth model curve for males P. volitans in the VASNP, Veracruz, Mexico.

Los valores individuales de $K_{N}$ formaron un intervalo de 0.0005 a 0.0016 , con una media de $0.0007 \pm 00016$; en las tablas 1 y 2 se observan los valores de $K_{N}$ de las clases de tallas de hembras y machos.

Tabla 1. Valores de $\mathrm{K}_{\mathrm{N}}$ para cada clase de talla de hembras de P. volitans en el PNSAV, Veracruz, México. Table $1 . K_{\mathbf{N}}$ values for each size class of $P$. volitans females in the VASNP, Veracruz, Mexico.

\begin{tabular}{cc} 
Clases de talla $(\mathrm{mm})$ & $\mathrm{Kn}$ \\
\hline 15.3 & 0.00083372 \\
25.3 & 0.00081748 \\
28.2 & 0.00074393 \\
31.8 & 0.00078481
\end{tabular}

Tabla 2. Valores de $\mathrm{K}_{\mathrm{N}}$ para cada clase de talla de machos de P. volitans en el PNSAV, Veracruz, México. Table $2 . K_{\mathbf{N}}$ values for each size class of $P$. volitans females in the VASNP, Veracruz, Mexico.

\begin{tabular}{cc} 
Clases de talla $(\mathrm{mm})$ & $\mathrm{Kn}$ \\
\hline 9.87 & 0.83726026 \\
17.95 & 0.94959147 \\
22.95 & 0.87762237 \\
26.95 & 0.81643315 \\
30.95 & 0.71889526 \\
33.95 & 0.77669568
\end{tabular}




\section{Análisis de contenido estomacal}

Se realizó el análisis del contenido estomacal a 97 individuos, de los cuales, 26 (26.8\%) fueron estómagos vacíos o regurgitados, y 71 (73.9\%) fueron estómagos con algún tipo de presa. Se identificaron doce ítems alimenticios pertenecientes a seis familias de peces, ordenadas de acuerdo con el criterio taxonómico de Nelson (2006). Se identificaron cuatro familias y cinco especies de crustáceos decápodos (Tabla 3). En el caso de las hembras, los peces fueron la presa más abundante representando 90.07\%, Haemulon aurolineatum (Cuvier, 1830) es la presa con mayor porcentaje IIR $(84.31 \%)$, se presentó únicamente en la clase de talla 1, ocupando más del $90 \%$. Los crustáceos ocupan el 9.92\% restante, siendo Xiphopenaeus kroyeri (Heller, 1862) la presa con mayor porcentaje IIR (5.24\%) estando presente en las primeras dos clases de talla (Tabla 3) (Figs. 10 y 11).

Los peces $(64.70 \%)$ fueron la presa más importante en los machos; $D$. bivittatum fue la presa principal con un porcentaje IIR de 44.01\% (Tabla 3), presentándose en las tallas mayores (clase de talla 6), el 35.3\% restante estuvo representado por crustáceos, siendo la principal presa X. kroyeri con in porcentaje IIR de 20.69\%, apareciendo en la clase de talla 1 (Tabla 3 y Figs. 10 y 11).

Tabla 3. Parámetros del contenido estomacal e ítems alimenticios identificados, índice numérico (\% $\mathrm{N})$, gravimétrico $(\% \mathrm{P})$, de ocurrencia (\%F) e índice de importancia relativa en porcentaje (\%IIR) de P. volitans en el PNSAV. Los ítems marcados con un asterisco $\left(^{*}\right)$ corresponden a crustáceos.

Table 3. Stomach content and identified food items parameters,), percent composition by number (\%N), percent composition by volume $(\% \mathrm{~V})$ and percent frequency of occurrence $(\% \mathrm{~F})$ and the percent index of relative importance (\% IRI) of P. volitans in the VASNP. Items marked with an asterisk $\left(^{*}\right)$ are crustaceans.

\begin{tabular}{|c|c|c|c|c|c|c|c|c|}
\hline \multirow[b]{2}{*}{ Presa } & \multicolumn{4}{|c|}{ Hembras } & \multicolumn{4}{|c|}{ Machos } \\
\hline & $\% \mathrm{~N}$ & $\% \mathrm{P}$ & $\% \mathrm{~F}$ & $\%$ IIR & $\% \mathrm{~N}$ & $\% \mathrm{P}$ & $\% \mathrm{~F}$ & $\% \operatorname{IIR}$ \\
\hline Haemulon aurolineatum (Cuvier, 1830) & 62.162 & 50.541 & 31.25 & 84.317 & 0 & 0 & 0 & 0 \\
\hline Ophidion josephi (Girard, 1858) & 2.702 & 2.303 & 6.25 & 0.749 & 0 & 0 & 0 & 0 \\
\hline Microspathodon chrysurus (Cuvier, 1830) & 0 & 0 & 0 & 0 & 25 & 16.386 & 25 & 20.693 \\
\hline Diplectrum bivittatum (Valenciennes, 1828) & 2.702 & 16.220 & 6.25 & 2.831 & 25 & 63.025 & 25 & 44.012 \\
\hline Parablennius marmoreus (Poey, 1876) & 2.702 & 1.175 & 6.25 & 0.5805 & 0 & 0 & 0 & 0 \\
\hline Achiridae (Rafinesque, 1815) & 2.702 & 4.560 & 6.25 & 1.086 & 0 & 0 & 0 & 0 \\
\hline Diogenidae * (Ortman, 1892) & 2.702 & 0.188 & 6.25 & 0.432 & 0 & 0 & 0 & 0 \\
\hline Squilla sp. * (Fabricius, 1787) & 2.702 & 4.701 & 6.25 & 1.107 & 0 & 0 & 0 & 0 \\
\hline
\end{tabular}




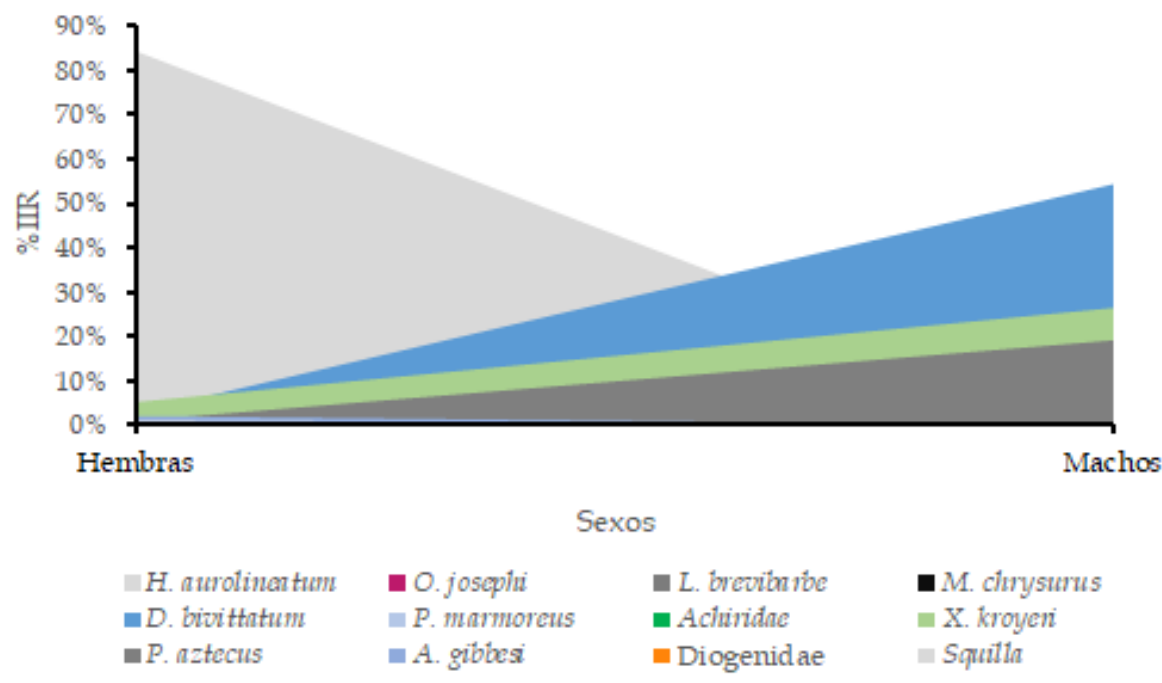

Fig. 10. Distribución de la dieta de P. volitans de acuerdo al \%IIR en hembras y machos, en el PNSAV, Veracruz, México. Fig. 10. Diet distribution of $P$. volitans females and males, according to the \%IIR in the VASNP, Veracruz, Mexico.

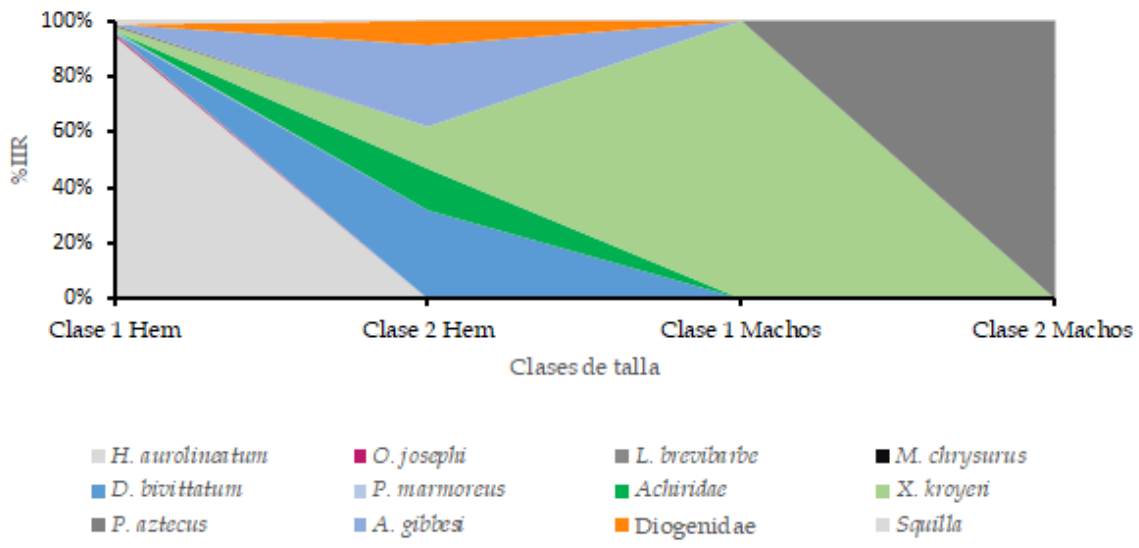

Fig. 11. Distribución de la dieta de P. volitans de acuerdo al \%IIR de clases de talla 1 y 2 en hembras y machos en el PNSAV, Veracruz.

Fig. 11. Diet distribution of $P$. volitans according to $\%$ IIR of size classes 1 and 2 in females and males in VASNP, Veracruz.

La comparación de dietas entre tallas se hizo únicamente con las clases de talla 1 y 2 para hembras y machos, debido a que fueron las tallas en las cuales se encontraron estómagos con alimento.

En la clase de talla 1 en hembras se observaron siete ítems, $H$. aurolineatum, O. josephi, L. brevibarbe y $P$. marmoreous, X. kroyeri, P. aztecus y el género Squilla; en la clase de talla 2 se registraron cinco ítems, $X$. kreoyeri, A. gibbesi, un organismo de la familia Diogenidae, D. bivittatum y un representante de la familia Achiridae. 
En las clases de talla 1, 2 se presentó un ítem (X. kroyeri y P. aztecus respectivamente), en las clases de talla mayores se presentó la preferencia por peces como D. bivittatum.

\section{DISCUSIÓN}

El crecimiento del pez león en el PNSAV, expresado a través de la relación talla-peso, exhibió un crecimiento de tipo alométrico en ambos sexos (Figs. 8 y 9), esto significa que se presenta un crecimiento acelerado en las primeras etapas de vida de los organismos, lo que es un comportamiento típico en el crecimiento de especies tropicales (Sparre y Venema, 1997).

Acorde con el presente estudio, autores como Darling et al. (2011), Sabido et al (2012), Sabido-Itzá et al. (2016), reportan un tipo de crecimiento alométrico para peces en el hábitat nativo, así como para hábitats invadidos, Barbour et al. (2011) y Sandel et al. (2015) calcularon valores de $b$ menores a 3 en hábitats de Carolina del Norte, Estados Unidos y Costa Rica respectivamente, lo que contrasta con lo reportado aquí. Díaz-Pérez et al. (2016), mencionan que estas variaciones pueden ser causadas por diversos factores como la ubicación geográfica, salud del arrecife y tipo de hábitat, sumado a factores bióticos como la alimentación, madurez sexual y tipo de crecimiento (Morales y González, 2010). En este sentido, el tipo de crecimiento observado en el presente estudio permite inferir que existe una condición física favorable que tiene como consecuencia el establecimiento de la especie en el PNSAV (Toledo-Hernández et al., 2014).

Así mismo, el bienestar somático de la población establecida en el PNSAV se ve reflejado en el factor de condición relativo $(\mathrm{Kn})$, que de acuerdo con los valores para hembras obtenidos $\left(0.0007 \pm 1.98 \times 10^{-5}\right)$, son similares a los reportados por Toledo-Hernández et al. (2014) para Puerto Rico $(0.008 \pm 0.0002)$, resaltando el hecho de que los valores obtenidos para machos se encuentran por arriba de los reportados por estos autores. Las diferencias encontradas para ambos sexos se pueden atribuir a las tallas registradas, así como a la pérdida de peso que sufren las hembras por efecto del desove presentado durante todo el ciclo anual (asincrónico) (Froese, 2006; Paz, 2016).

De acuerdo con Santander-Monsalvo et al. (2012), la presencia del pez león en el PNSAV es reciente, registrando en principio solo su abundancia y sitios de avistamiento, en consecuencia los estudios sobre crecimiento y relación LP-W para el PNSAV no se han abordado, por lo que este trabajo proporciona información básica para la comprensión del crecimiento y su relación con la dinámica poblacional de $P$. volitans en este sistema arrecifal, permitiendo establecer la pauta para el manejo y control del crecimiento de esta especie en el área de estudio.

Por otra parte, las tallas promedio registradas en ambos sexos ( $185 \pm 6.7 \mathrm{~mm}$ y $183 \pm 15.8 \mathrm{~mm})$ son parecidas a lo reportado por Sabido-Itzá et al. (2016), para el Caribe mexicano, comprendidas entre $187.8 \pm 34.8 \mathrm{~mm}$ en 2011 y $219.7 \pm 55.9 \mathrm{~mm}$ en 2012, mientras que en Costa Rica se reportan 187士57 mm de talla promedio (Sandel et al., 2015); y para Colombia, Hernández-Abello et al. (2015) registraron $180 \pm 53 \mathrm{~mm}$. Tomando como referencia la talla mínima de madurez calculada para el Atlántico occidental de 175 a $189 \mathrm{~mm}$ (Morris, 2009; Gardener et al., 2015), los IGS promedio de hembras (0.0076) y machos (0.0057) obtenidos en este estudio, se observa que éstos son menores a los reportados por Gardener et al. (2015) de 1.96 y 0.0301 respectivamente. Estas diferencias pueden estar relacionadas con la temperatura del agua, como lo mencionan Gardener et al. (2015) y Paz (2016), quienes dicen que entre más estable sea la temperatura del agua, existe un mayor éxito reproductivo, reflejándose en los valores de IGS.

Los datos obtenidos sugieren que la población de $P$. volitans se encuentra ya establecida en el PNSAV de manera permanente, debido a la presencia de juveniles y adultos en el área de 
estudio, esto concuerda con lo mencionado por Sabido-Itzá et al. (2016); la distribución de tallas observadas indica que el PNSAV es un área importante en el reclutamiento del pez león, ya que la mayor abundancia se registra entre 147 y $164 \mathrm{~mm}$ en hembras y 130 a $149 \mathrm{~mm}$ en machos, siendo éstos organismos inmaduros (Vásquez-Yeomans et al., 2011).

Los resultados correspondientes a la dieta indican que el pez león se comporta como un depredador oportunista dentro del área de estudio, ya que se alimenta principalmente de peces teleósteos y en menor medida de crustáceos, lo que coincide con los resultados obtenidos en el Parque Nacional Arrecife Alacranes, Yucatán (79\% y 21\% de ocurrencia), Parque Nacional Arrecifes de Cozumel, Yucatán (58.8\% y 34.3\% de ocurrencia), Holguín, Cuba (99.11\%IIR para teleósteos) y en Colombia (92.5\% y 7.5\% de ocurrencia) (Muñoz-Escobar et al., 2012; Quijano-Puerto et al., 2013; Bogdanoff et al., 2018; Reynaldo et al., 2019 ).

Se observó que las hembras presentan como presa con mayor aporte a H. aurolineatum, ésta especie se encuentra distribuida principalmente en el Atlántico occidental, habitando en pastos marinos y arrecifes de coral (Robins et al., 1986), y es abundante en el PNSAV (Martínez y Camacho, 2007; Pérez y Vargas, 2008). Ayala-Rodríguez et al. (2016) reportan a la familia Haemulidae como la que aporta mayor abundancia de larvas en el PNSAV (47.62\%), siendo $H$. aurolineatum la especie que contribuyó con $28.97 \%$, sugiriendo una población de reclutas y adultos abundante (Doherty et al., 2004), lo cual coincide con lo observado en el presente estudio, ya que los individuos encontrados en los estómagos fueron juveniles, lo que puede impactar de manera importante el reclutamiento de esta especie. Debido a sus antecedentes de ser un organismo generalista (Muñoz-Escobar y Gil-Agudelo, 2012; Quijano-Puerto et al., 2013; Eddy et al., 2016), el pez león consume preferentemente a $H$. aurolienatum debido a que esta especie es el recurso más disponible.

Para el caso de los machos, los peces teleósteos resultaron ser también la presa con mayor importancia, siendo D. bivittatum la más importante. Morán-Silva et al. (2017) mencionan que en la pesca industrial de camarón café en la porción central de la costa veracruzana, $D$. bivittatum es una de las especies que registran mayor abundancia en la pesca de descarte, si se suma la depredación del pez león, esta especie también podría verse afectada en su reclutamiento, ya que el promedio en las tallas de captura incidental registrada es $7.84 \pm 2.10 \mathrm{~cm}$, mientras que la talla promedio en el presente estudio es $6.4 \mathrm{~cm}$, la que está por debajo de la $\mathrm{L}_{\max }$ estimada de $25.0 \mathrm{~cm}$ (Robins et al., 1986).

Respecto a los crustáceos, X. kroyeri es la presa con mayor \%IIR en hembras y machos. Este crustáceo decápodo es de importancia económica en el sureste del golfo de México, principalmente en las costas de Campeche (Wakida, 2005). Esta especie no ha sido reportada en el PNSAV como un organismo importante económicamente, sin embrago, Geissert y Enríquez (2011), reportan su presencia en el arrecife Hornos.

Se puede concluir que el pez león rojo se ha establecido exitosamente dentro del PNSAV, debido a que las tallas encontradas son representativas de la historia de vida de la especie, aún con el sesgo que se pueda llegar a presentar por el arte de pesca utilizado en su captura (arpón hawaiano). $P$. volitans es una especie deprtedadora oportunista, que consume especies de peces y crustáceos disponibles en el PNSAV, sin embargo, es necesaria mayor información sobre $D$. bivittatum, X. kroyeri y H. aurolineatum para poder evaluar de forma precisa el impacto del pez león en estas poblaciones. Es importante identificar procesos ecológicos que puedan limitar la invasión de ésta especie al PNSAV, que se vean reflejadas en estrategias de manejo y control de $P$. volitans. 


\section{AGRADECIMIENTOS}

Los autores agradecen el apoyo de la Comisión Nacional de Áreas Naturales Protegidas (CONANP) y a la cooperativa de Antón Lizardo por facilitar los ejemplares utilizados en el presente estudio.

\section{REFERENCIAS}

1. Aguilar-Perera A., Perera-Chan L. y Quijano-Puerto L., 2013. Record body size for the red lionfish, Pterois volitans (Scorpaeniformes), in the Southern Gulf of Mexico. Gulf and Caribbean Research, 25: 121-123. DOI: 10.18785/gcr.2501.10

2. Albins M.A. y Hixon M.A., 2008. Invasive Indo-Pacific lionfish Pterois volitans reduce recruitment of Atlantic coral-reef fishes. Marine Ecology Progress Series, 367: 233-238. DOI: https://doi.org/10.3354/meps07620

3. Ayala-Rodríguez G.A., Ordóñez-López U., Meiners C. y Marín-Hernández M., 2016. Listado taxonómico, aspectos ecológicos y biogeográficos de las larvas de peces del Sistema Arrecifal Veracruzano, Suroeste del Golfo de México (junio 2011-junio 2013). Revista de Biología Marina y Oceanografía, 51(2): 255-264. DOI: http://dx.doi.org/10.4067/S0718-19572016000200004

4. Barbour A.B., Allen M.S., Frazer T.K. y Sherman K.D., 2011. Evaluating the potential efficacy of invasive lionfish (Pterois volitans) removals. PloS one, 6: e19666. DOI: 10.1371/journal.pone.0019666

5. Bhattacharya C.G. (1967). A simple method of resolution of a distribution into gaussian components. Biometrics, 23(1): 115-135. DOI: 10.2307/2528285

6. Bogdanoff A.J., Mostowy J., Peake J., Layman C.A., Brito A.B., Gonzalez C.B., Hernández N.P., Martinez D.T.G., Bravo M.R.X. y Morris J.A., 2018. A brief description of invasive lionfish (Pterois sp.) diet composition in the Arrecifes de Cozumel National Park. Food Webs, 17: e00104. DOI: https://doi.org/10.1016/j.fooweb.2018.e00104

7. Darling E.S., Green S.J., O’Leary J.K. y Côté I.M., 2011. Indo-Pacific lionfish are larger and more abundant on invaded reefs: a comparison of Kenyan and Bahamian lionfish populations. Biological invasions, 13: 2045-2051. DOI: https://doi.org/10.1007/s10530-011-0020-0

8. Diario Oficial de la Federación (DOF), 1992. Decreto por el que se declara área natural protegida con el carácter de Parque Marino Nacional, la zona conocida como Sistema Arrecifal Veracruzano, ubicada frente a las Costas de los municipios de Veracruz, Boca del Río y Alvarado del estado de Veracruz Llave, con superficie de 52,238-91-50 hectáreas. Diario Oficial de la Federación, México. Disponible en: http:/ / dof.gob.mx/nota_detalle.php?codigo=5280548\&fecha=29/11/2012

9. Doherty P.J., Dufour V., Galzin R., Hixon M.A., Meekan M.G. y Planes S., 2004. High mortality during settlement is a population bottleneck for a tropical surgeonfish. Ecology, 85: 2422-2428. DOI: https://doi.org/10.1890/04-0366

10. Eddy C., Pitt J., Morris J.A. Jr., Smith S., Goodbody-Gringley G. y Bernal D., 2016. Diet of invasive lionfish (Pterois volitans and P. miles) in Bermuda. Marine Ecology Progress Series, 558: 193206. DOI: https://doi.org/10.3354/meps11838 
11. Edelist D., Rilov G., Golani D., Carlton J. T. y Spanier E., 2013. Restructuring the sea: profound shifts in the world's most invaded marine ecosystem. Diversity and distributions, 19: 69-77.

DOI: https://doi.org/10.1111/ddi.12002

12. Fishelson L., 1997. Experiments and observations on food consumption, growth and starvation in Dendrochirus brachypterus and Pterois volitans (Pteroinae, Scorpaenidae). Environmental Biology of Fishes, 50: 391-403. DOI: https://doi.org/10.1023/ A:1007331304122

13. Froese R., 2006. Cube law, condition factor and weight-length relationships: history, metaanalysis and recommendations. Journal of Applied Ichthyology, 22(4): 241-253. DOI: https://doi.org/10.1111/j.1439-0426.2006.00805.x

14. Gardner P.G., Frazer T.K., Jacoby C.A. y Yanong R.P.E, 2015. Reproductive biology of invasive lionfish (Pterois spp.). Frontiers in Marine Science, 2: 1-10. DOI: https://doi.org/10.3389/fmars.2015.00007

15. Geissert D.K. y Enríquez E.F., 2011.Geomorfología. En: Comisión Nacional para el Conocimiento y Uso de la Biodiversidad (CONABIO), La Biodiversidad en Veracruz: estudio de estado, Vol. I, pp. 53-68. México, Comisión Nacional para el Conocimiento y Uso de la Biodiversidad, Gobierno del Estado de Veracruz, Universidad Veracruzana, Instituto de Ecología, A. C. México.

16. Gompertz B., 1825. On the nature of the function expressive of the law of human mortality, and on a new mode of determining the value of life contingencies. Philosophical Transactions of the Royal Society of London, 115: 513-583.

17. Hernández-Abello J.M., García-Urueña R.P. y Acero A., 2015. Estructura de tallas y preferencia al sustrato del pez león (Pterois volitans) (Scorpaeniformes: Scorpaenidae) en Santa Marta (Colombia). Acta Biológica Colombiana, 20(2): 175-182. DOI: http://dx.doi.org/10.15446/abc.v20n2.41611

18. Korschgen L., 1980 Procedures for food habits analyses. En: Schemnitz S.D. (Ed.), Wildlife Management Techniques Manual, pp. 113-119. Washington, The Wildlife Society.

19. Krutak P.R. 1997. Petrography and provenance of siliciclastic sediments, Veracruz-Anton Lizardo Reefs, Mexico. En: J. San-Joon Jan y Hi-Il, Y (Eds.), Paleoceanography and Paleoclimatology in the Northwest Pacific Region. Special Issue 3, Ocean Research, 19(3): 231-243.

20. Kuiter R.H. y Tonozuka T., 2001. Pictorial guide to Indonesian reef fishes. Part 3. Jawfishes Sunfishes, Opistognathidae - Molidae. Zoonetics, Australia.

21. Lugo-Castro U.N., Lázaro-López O.B., Alanís-Méndez J.L. y Limón F., 2018. Predicción del impacto del pez león (Pterois sp.) en áreas marinas protegidas del Atlántico usando modelos de nicho ecológico. VII Congreso Internacional Biológico y Agropecuario, Tuxpan, Veracruz, Mexico. Tuxpan, Veracruz, México. Realizado del 3 al 5 de octubre de 2018. DOI: 10.13140/RG.2.2.13061.40164 
22. Martínez J.A.H. y Camacho B.O., 2007. Ictiofauna del arrecife artificial "Ex - Cañonero C-50, General Vicente Riva Palacio" en el Sistema Arrecifal Veracruzano, México. En: Granados-Barba A., Abarca-Arenas L.G. y Vargas-Hernández J.M. (Eds.), Investigaciones Científicas en el Sistema Arrecifal Veracruzano, pp. 209-220. México, Universidad Autónoma de Campeche.

23. Morales M. y González L.W., 2010. Edad y crecimiento del pez Haemulon steindachneri (Perciformis: Haemulidae) en el suroeste de la isla de Margarita, Venezuela. Revista de Biología Tropical, 58(1): 299-310.

24. Morán-Silva A., Chávez-López R., Jiménez-Badillo M.A., Cházaro-Olvera S., Galindo-Cortes G. y Meiners-Mandujano C.G., 2017. Análisis de la comunidad de peces de descarte en la pesca de arrastre de camarón (temporada de lluvias 2013) en la zona centro-sur del litoral veracruzano, México. Revista de biología marina y Oceanografía, 52: 551-566. DOI: 10.4067/S071819572017000300012

25. Morris J.A. Jr., 2009. The biology and ecology of the invasive Indo-Pacific lionfish (tesis doctoral, Graduate Faculty of North Carolina State University, USA).

26. Morris J.A. y Akins J.L., 2009. Feeding ecology of invasive lionfish (Pterois volitans) in the Bahamian archipelago. Environmental Biology of Fishes, 86: 389-398. DOI: https://doi.org/10.1007/s10641-009-9538-8

27. Muñoz-Escobar L. y Gil-Agudelo D.L., 2012. Composición dietaria del pez león, Pterois volitans (Pisces: Scorpaenidae), en Santa Marta y el Parque Nacional Natural Tayrona. Boletín de Investigaciones Marinas y Costeras-INVEMAR, 41(2): 471-477.

28. Nelson J.S., 2006. Fishes of the world. Nueva York, John Wiley \& Sons.

29. Olaya-Nieto C., Soto-Fernández P. y Barrera-Chica J., 2009. Hábitos alimentarios de la mayupa (Sternopygus macrurus, Bloch \& Schneider, 1801) en el río Sinú, Colombia. Revista. MVZ Córdoba 14(3): 1787-1795. DOI: https://doi.org/10.21897/rmvz.33묘

30. Paz Y.A.R., 2016. Biología reproductiva del pes león (Pterois volitans, Linnaeus, 1758) (Scorpaenidae) en el sector oeste de la reserva de la Biosfera Baconao , Cuba (tesis de maestría, CICIMAR, Instituto Politécnico Nacional, La Paz, Baja California Sur, México).

31. Pérez H.E. y Vargas J.M.H. 2008. Caracterización ecológica y monitoreo del Parque Nacional Sistema Arrecifal Veracruzano: Primera Etapa. Universidad Veracruzana. México, Centro de Ecología y Pesquerías. Bases de datos SNIB-CONABIO, proyecto No. DM002. Disponible en http://www.conabio.gob.mx/institucion/proyectos/resultados/InfDM002.pdf

32. Pinkas L., Oliphant M.S. e Iverson I.L.K., 1970. Food habits of albacore, bluefin tuna, and bonito in California waters. Fish Bulletin, 152: 1-105.

33. Quijano-Puerto L., Perera-Chan L., Aguilar-Perera A. y Tuz Sulub A., 2013. Dieta del pez león rojo, Pterois volitans, en el Parque Nacional Arrecife Alacranes, Sureste del Golfo de México. Proceedings of the Gulf and Caribbean Fisheries Institute, 65: 333-337. Disponible en http:/ /aquaticcommons.org/21517/ 
34. Reynaldo E.C., Veja A.T., Veja M.E.C., Fernández A.V., Cruz J.R., Córdova E.G. Cruz P.R., 2019. Preferencias tróficas de Pterois volitans (Socrpaenidae) en el área costera de Holguín, Cuba. Novitates Caribaea, 13: 1-12. DOI: https://doi.org/10.33800/nc.v0i13.188

35. Ricker W.E., 1975. Computation and interpretation of biological statistics of fish populations. Bulletin of the Fisheries Research Board of Canada, 191. Ottawa, Canada, Fisheries and Marine Service.

36. Robins C.R., Ray G. C., y Douglass J., 1986. A field guide to Atlantic coastal fishes of North America. Boston, EE. UU: Houghton Mifflin Company.

37. Sabido M.M.I, Gómez-Poot J.M., Medina A.Q. García M.C.R. y Hadad W.L., 2012. Dinámica poblacional del pez León (Pterois volitans) en el Parque Nacional Arrecifes de Xcalak (PNAX), Quintana Roo, Caribe mexicano. Proceedings of the Gulf and Caribbean Fisheries Institute, 64: 6774. Disponible en http://aquaticcommons.org/21324/

38. Sabido-Itzá M., Medina-Quej A., Navarrete A., Gómez-Poot J., y García-Rivas M., 2016. La estructura de tallas como evidencia del establecimiento de Pterois volitans (Scorpaeniformes: Scorpaenidae) en el sur del Caribe mexicano. Revista de Biología Tropical, 64: 353-362. DOI: https://dx.doi.org/10.15517/rbt.v64i1.18943

39. Salas-Pérez J.J. y Granados-Barba A., 2008. Oceanographic characterization of the Veracruz reefs system. Atmósfera, 21(3): 281-301.

40. Sandel V., Martínez-Fernández D., Wangpraseurt D. y Sierra L., 2015. Ecology and management of the invasive lionfish Pterois volitans/miles complex (Perciformes: Scorpaenidae) in Southern Costa Rica. Revista de Biología Tropical, 63: 213-221. DOI: https://doi.org/10.15517/rbt.v63i1.14749

41. Santander-Monsalvo J., López-Huerta I., Aguilar-Perera A. y Tuz-Sulub A., 2012. First record of the red lionfish (Pterois volitans [Linnaeus, 1758]) off the coast of Veracruz, Mexico. BioInvasions Records, 1: 121-124. DOI: http://dx.doi.org/10.3391/bir.2012.1.2.07

42. Schultz E. T., 1986. Pterois volitans and Pterois miles: two valid species. Copeia, 3: 686-690.

43. Sparre P. y Venema S.C. (Eds.), 1997. Introducción a la evaluación de recursos pesqueros tropicales. Parte 1. Manual. FAO Documento técnico de Pesca, Vol. 306, Roma, FAO.

44. Toledo-Hernández C., Vélez-Zuazo X., Ruiz-Díaz C. P., Patricio A. R., Mège P., Navarro M., Sabat A. M., Betancur-R R. y Papa R., 2014. Population ecology and genetics of the invasive lionfish in Puerto Rico. Aquatic Invasions, 9(2): 227-237. DOI: http://dx.doi.org/10.3391/ai.2014.9.2.12

45. Vásquez-Yeomans L., Vega-Cendejas M.E., Montero J.L. y Sosa-Cordero E. 2011. High species richness of early stages of fish in a locality of the Mesoamerican Barrier Reef System: a small-scale survey using different sampling gears. Biodiversity and Conservation, 20: 2379-2392. DOI: https://doi.org/10.1007/s10531-011-9990-6

46. Villaseñor-Derbez J.C. y Herrera-Pérez R., 2014. Brief description of prey selectivity and ontogenetic changes in the diet of the invasive lionfish Pterois volitans (Actinopterygii, Scorpaenidae) in the Mexican Caribbean. Pan-American Journal of Aquatic Sciences, 9: 131-135. 
47. Wakida A.T.K., 2005) Análisis de la captura incidental en la pesquería ribereña del camarón siete barbas Xiphopenaeus kroyeri en las costas de Campeche, México. Proceedings of the Gulf and Caribbean Fisheries Institute, 56: 583-591. Disponible en http://aquaticcommons.org/13931/

48. Wakida-Kusunoki A.T. y Amador L.E.A., 2015. First record of the red lionfish, Pterois volitans, on the coast of Tabasco, Mexico. Hidrobiológica, 25(2): 307-309.

49. Yáñez-Arancibia A., 1986. Ecología de la zona costera: análisis de siete tópicos. México, A.G.T. Editor.

BIOCYT Biología, Ciencia y Tecnología, se encuentra actualmente indexada en
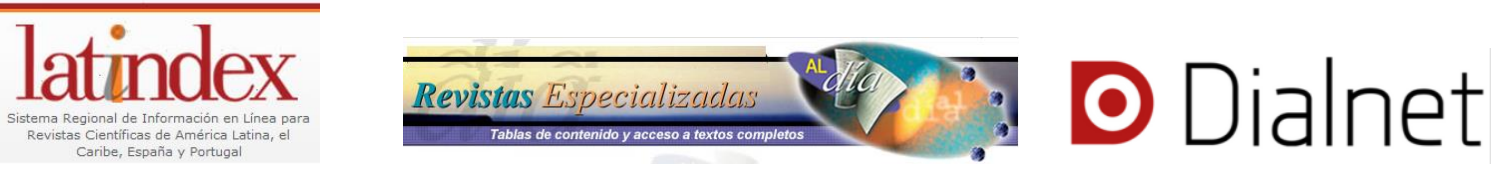

\section{Actualidad Iberoamericana}
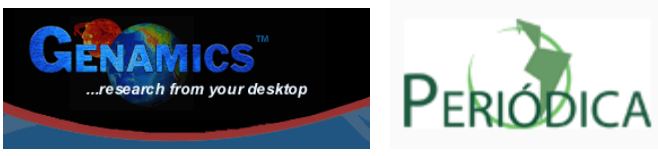

\section{ERIHPLUS \\ EUROPEAN REFERENCE INDEX FOR THE
HUMANITIES AND SOCIAL SCIENCES}

alojada en los repositorios

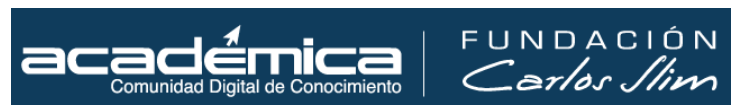

Joumals for Irree
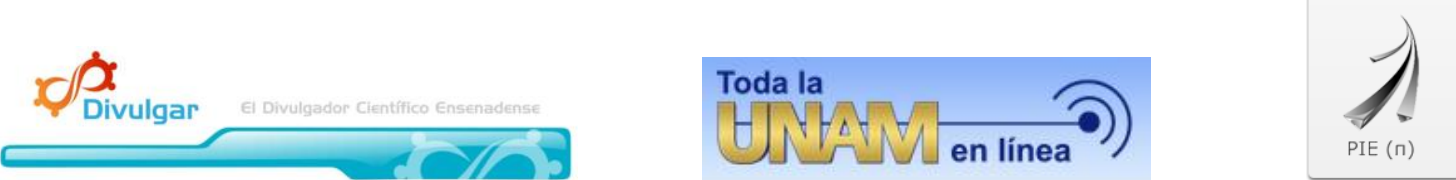

Hepositorio Institucional Científico y Académico 
en bases electrónicas de bibliotecas
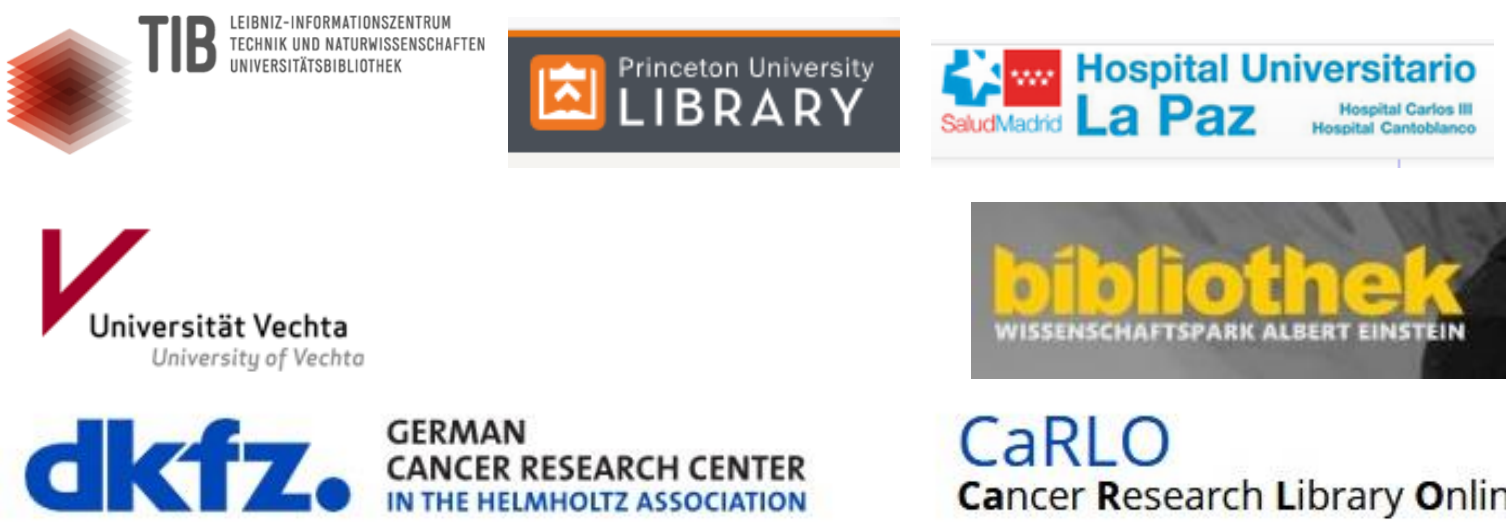

\section{CaRLO}

Cancer Research Library Online

y en buscadores académicos
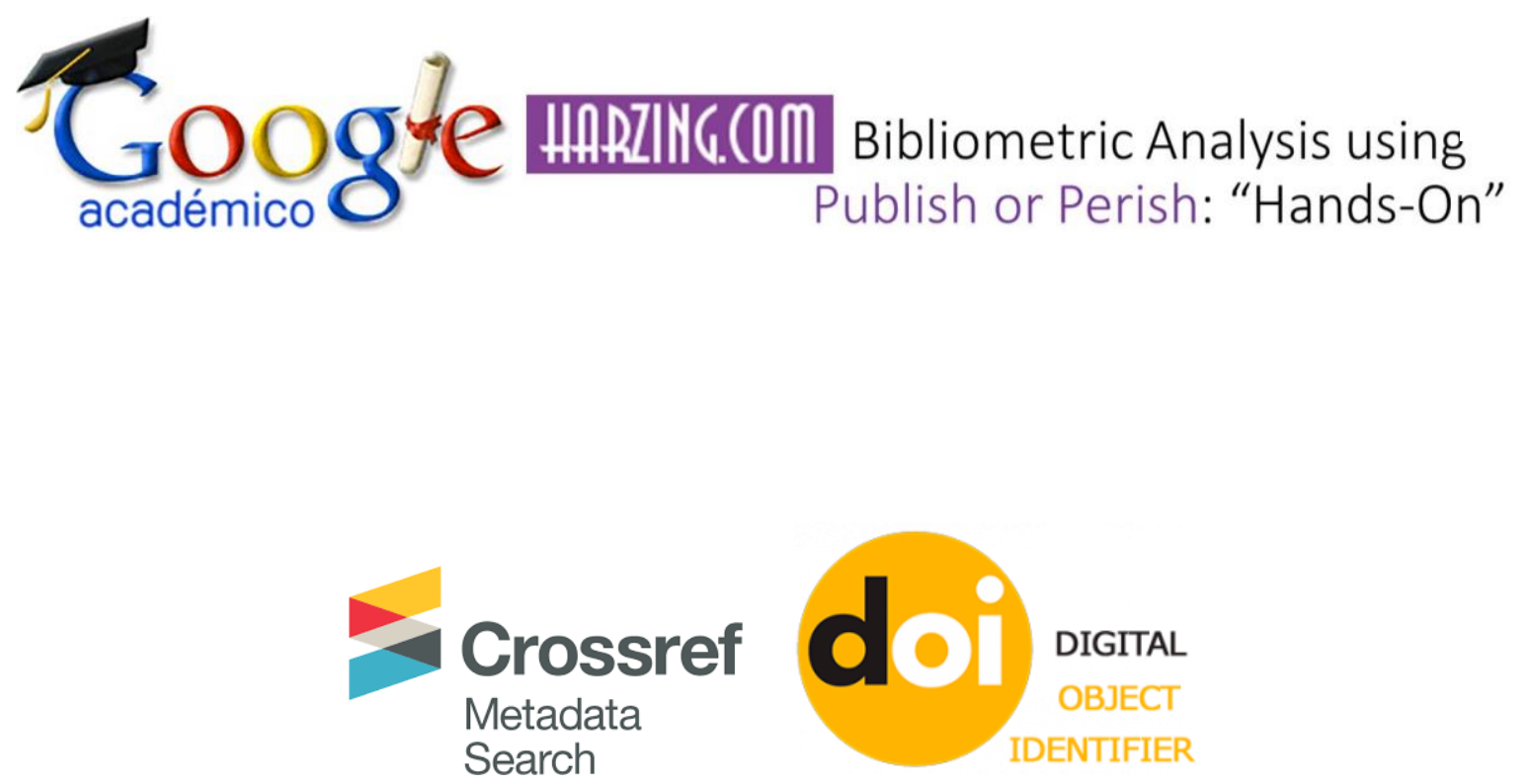